\title{
Dissecting the genetic architecture of quantitative traits using genome-wide identity-by-descent sharing among full-sibs
}

\author{
Antoine Fraimout* ${ }^{11}$,Zitong Li $^{1}$, Mikko J. Sillanpää ${ }^{2}$, Pasi Rastas ${ }^{1,3}$ \& Juha Merilä ${ }^{1,4}$ \\ ${ }^{1}$ Ecological Genetics Research Unit, Organismal and Evolutionary Biology Research Programme, Faculty of Biological and Environmental \\ Sciences, FI-00014 University of Helsinki, Finland \\ ${ }^{2}$ Research Unit of Mathematical Sciences, FI-90014 University of Oulu, Finland \\ ${ }^{3}$ Institute of Biotechnology, FI-00014 University of Helsinki \\ ${ }^{4}$ Division of Ecology and Biodiversity, Faculty of Science, The University of Hong Kong, Hong Kong SAR \\ *Corresponding author: fraimout.antoine@gmail.com
}

\begin{abstract}
Additive and dominance genetic variances underlying the expression of quantitative traits are important quantities for predicting short-term responses to selection, but they are notoriously challenging to estimate in most wild animal populations. Using estimates of genome-wide identity-by-descent (IBD) sharing from autosomal SNP loci, we estimated quantitative genetic parameters for traits known to be under directional natural selection in nine-spined sticklebacks (Pungitius pungitius) and compared these to traditional pedigreebased estimators. Using four different datasets, with varying sample sizes and pedigree complexity, we further assessed the performance of different Genomic Relationship Matrices (GRM) to estimate additive and dominance variance components. Large variance in IBD relationships allowed accurate estimation of genetic variance components, and revealed significant heritability for all measured traits, with negligible dominance contributions. Genome-partitioning analyses revealed that all traits have a polygenic basis and are controlled by genes at multiple chromosomes. The results demonstrate how large full-sib families of highly fecund vertebrates can be used to obtain accurate estimates quantitative genetic parameters to provide insights on genetic architecture of quantitative traits in non-model organisms from the wild. This approach should be particularly useful for studies requiring estimates of genetic variance components from multiple populations as for instance when aiming to infer the role of natural selection as a cause for population differentiation in quantitative traits.
\end{abstract}

Keywords: quantitative genomics, heritability, identity-by-descent, Pungitius, relatedness, dominance 


\section{Introduction}

Ever since Fisher's pioneer work on the genetic resemblance between relatives (Fisher, 1918) the decomposition of phenotypic variance into its genetic and non-genetic components has been at the center of quantitative genetics. Accurate estimation of the genetic variance components underlying quantitative trait variability is important to our ability to predict the association between human diseases and genetic variants (Tenesa \& Haley, 2013), to understand the genetic architecture of complex traits and to resolve equations of short-term response to selection (Lynch \& Walsh, 1998). The latter has been particularly important in the field of evolutionary biology to understand how species adapt to their environment, and how genetic variation is shaped throughout evolution (Lynch \& Walsh, 1998; Walsh \& Lynch, 2018). A central parameter in quantitative genetics is the narrowsense heritability $\left(h^{2}\right)$ which describes the amount of phenotypic variation attributable to genetic inheritance $\left(h^{2}=\mathrm{V}_{\mathrm{A}} / \mathrm{V}_{\mathrm{P}}\right.$; where $\mathrm{V}_{\mathrm{A}}$ is the additive genetic variance and $\mathrm{V}_{\mathrm{P}}$ the total phenotypic variance; Falconer \& Mackay, 1996). The breeder's equation $\left(R=h^{2} S\right.$; Lush, 1943) defines the response to selection $R$ as the product of a trait's heritability and the selection coefficient $S$. Therefore, accurate prediction of changes in a trait mean value over one generation of selection directly depends on how well the additive genetic variance is estimated and, ultimately, predictive models of phenotypic evolution rely on accurate estimation of genetic variance.

Traditionally, heritability estimation requires measures of continuous traits among individuals of known relatedness, using specific crossing designs in the laboratory, or pedigree information gathered from natural populations (Lynch \& Walsh, 1998). In both cases, establishing the degree of relatedness between individuals is logistically challenging because most non-model organisms are not amenable to laboratory rearing or because building sufficiently large pedigrees requires extensive, and often long-term, work in the wild. Consequently, our understanding of the genetic architecture of ecologically important traits in natural populations has long been restricted to those organisms from which sufficient relatedness information can be collected with relative ease. Furthermore, classical methods in quantitative genetics rely on the expected genetic relationship among related individuals rather than on the realized proportion of the genome that they share identically-by-descent (IBD), and thus, they are prone to inaccuracy in the estimation of genetic variances (Visscher, 2009). However, the genomic era has provided researchers access to an ever-increasing number of molecular markers, enabling fine mapping of genomic regions linked to variation in quantitative traits. In the context of relatedness studies, this abundance of markers allows for more accurate estimation of the probability of IBD sharing, and the identification of actual shared genomic regions between individuals. These advances in quantitative genomics have been well explored in the fields of human and medical genetics (Visscher et al.,, 2006, 2007; Manolio et al.,, 2009; Visscher, 2009; Powell et al.,, 2010; Speed \& Balding, 2015), animal and plant breeding (e.g. Edwards \& Batley, 2010; Hayes \& Goddard 2010; Sinclair-Waters et al., 2020), but relatively rarely in evolutionary ecology (e.g., Sillanpää, 2011; Thompson, 2013; Gienapp et al., 2017) and even less so in empirical context (but see Robinson et al. 2013, Perrier et al., 2018, Gienapp et al., 2019, Gervais et al. 2020, Duntsch et al. 2020, De la Cruz et al. 2020). This in spite the fact that studies on the probabilities of IBD among pedigreed individuals trace back far in the genetics of heredity (Malécot, 1948) and that molecular approaches to heritability estimation have been proposed over two decades ago (Ritland 1996; Mousseau et al. 1998 ; Ritland 2000).

An appealing prospect of quantitative genomics applied to natural populations is to take advantage of the variation in realized (i.e., actual) IBD resulting from within family segregation to estimate additive and dominance components of variance among sets of closely related individuals. This approach was first used to estimate heritability of height from human full-sibs, circumventing assumptions regarding environmental effects on phenotypic variation (i.e.; the topical "nature 
vs. nurture" bias; Visscher et al., 2006). Results from Visscher et al. (2006) revealed that realized IBD sharing among full-sibs can vary substantially around the theoretical expectation, and that this variance allows accurate estimation of additive genetic variance. Integrating this approach to the study of natural populations (viz. non-humans) could improve our understanding of the genetic basis of ecologically important traits, and more importantly, provide insight into the adaptive potential of natural populations facing rapid environmental changes (Gienapp et al. 2017). In practice, IBD sharing information can be used to estimate variance components of quantitative traits by integrating the additive genetic or dominance Genomic Relationship Matrix (GRM) in the animal model (Kruuk, 2004). In the animal model, additive genetic (co)variances among a set of individuals are included as a random effect through the additive genetic relationship matrix $\boldsymbol{A}$. The elements of $\boldsymbol{A}$ describe the degree of relatedness between any two individuals $i$ and $j$ such that $\boldsymbol{A}_{\mathrm{ij}}=2 \Theta_{i j}$ (where $\Theta$ corresponds to the coefficient of coancestry). Following theoretical expectations, $\Theta_{i j}$ for full siblings is 0.25 and their relatedness is therefore 0.50 . By replacing the elements of $\boldsymbol{A}$ with the realized, rather than expected IBD proportion between individuals, it is therefore possible to include genomic relatedness in the estimation of variance components through the animal model.

Despite the numerous prospective applications, utilization of genomic relatedness to the study of evolutionary potential in the wild is still uncommon (see above) and few studies have formally addressed the performance of GRM vs. pedigree approaches (but see Perrier et al., 2018). Moreover, compared to pedigree-based methods, animal models using IBD sharing information can potentially provide more accurate estimates of other important variance components, such as dominance genetic variance (Nishio \& Satoh, 2014; Granato et al., 2018). Although there is evidence to suggest that fitness-related traits might display more dominance variance than other traits (Crnokrak \& Roff, 1995; Merilä \& Sheldon, 1999), to the best of our knowledge, no study has made attempt to use genomic relatedness among full-sibs to estimate dominance variance in any trait. Full-sib family data should be particularly well suited for this, because an estimation of dominance variance is based solely on occurrence of such pairs of related individuals who can inherit with any probability the same two copies of alleles IBD from their ancestors appearing earlier in the pedigree. Full-sibs naturally fulfill this requirement and indeed with very high probability. To this date, the contribution of dominance genetic variance in the genetic architecture of quantitative traits is still unclear and requires additional investigation (Class \& Brommer, 2020).

The main objective of this study was to explore the utility of Visscher et al.'s (2006) idea to use full-sib data to estimate quantitative genetic parameters for ecologically important traits by using the nine-spined stickleback (Pungitius pungitius) as a model system. The species is particularly suitable for this purpose for three reasons: (i) it can be raised in laboratory conditions allowing standardization of environmental influences on trait values and (ii) its relatively high fecundity allows generating large GRMs between fullsibs. Furthermore, (iii) given the relatively small genome size of this species (Varadharajan et al., 2019; Kivikoski et al., 2020a) the variance in genome-wide IBD should be higher than for instance in humans (Xu, 2006), which in turn should provide high statistical power to infer magnitudes of additive and dominance contributions variance in quantitative traits (Visscher et al., 2006; Xu, 2006; Visscher, 2009). With focus on three phenotypic traits known to be subjected to directional selection in P. pungitius (Karhunen et al., 2014), we used estimates of genome-wide IBD sharing among large number of individuals to calculate SNPheritability (hereafter, heritability), additive and dominance variances for all traits, and compared them to estimates obtained from traditional pedigree-based animal models. Using a genome-partitioning approach (Visscher et al., 2007; Yang et al., 2011) we further investigated the potential polygenic basis of the focal traits and how different chromosomes contributed to their phenotypic variance. Our findings suggest that the 
utilized quantitative genomic approach based on fullsib families provides a cost-effective means to obtain material for quantitative genetic studies requiring data from multiple wild populations, such as those needed to infer role of natural selection in explaining observed degree of population differentiation (e.g. Karhunen et al. 2014; Leinonen et al. 2013).

\section{Material and methods}

\section{Study species}

The nine-spined stickleback is a small teleost fish species with a circumpolar distribution spanning across the northern hemisphere (Wootton, 1976). Ancestral marine $P$. pungitius populations have colonized multiple freshwater ponds wherein they have been isolated since the end of the last glaciation (Shikano et al., 2010; Teacher et al., 2011; Bruneaux et al., 2013). Pond populations have repeatedly evolved similar phenotypes - including gigantism (Herczeg et al., 2009a), faster growth rates (Herczeg et al., 2012), bold and aggressive personalities (Herczeg et al., 2009b) and reduction of the pelvic apparatus (Shikano et al., 2013; Kemppainen et al., 2020) - making them interesting models for evolutionary biology research (Merilä, 2013). Common garden and quantitative genetic studies indicate that there is significant genetic differentiation in the mean values of different quantitative traits (Herczeg et al., 2009b; Ab Ghani et al., 2012; Karhunen et al., 2014; Li et al., 2019), but little is known about trait heritabilities within populations (but see Shimada et al., 2011).

\section{Cross and fish husbandry}

Four different datasets were used in this study. Three of them consisted of $F_{2}$ generation inter-population crosses (hereafter " $F_{2}$ crosses") and one dataset corresponded to $F_{1}$ generation, lab-reared offspring from a wild marine population (hereafter "Helsinki crosses").

For the $\mathrm{F}_{2}$ crosses data, three inter-population crosses were produced by mating three different females from a marine population (Helsinki; $60^{\circ} 13^{\prime} \mathrm{N}, 25^{\circ} 11^{\prime} \mathrm{E}$ ) and three males from three different freshwater ponds:
Rytilampi (Finland, 66 $23^{\prime} \mathrm{N}, 2^{\circ} 19^{\prime} \mathrm{E}$ ), Pyöreälampi (Finland, $66^{\circ} 15^{\prime} \mathrm{N}, 29^{\circ} 26^{\prime} \mathrm{E}$ ) and Bynästjärnen (Sweden, $\left.64^{\circ} 27^{\prime} \mathrm{N}, 19^{\circ} 26^{\prime} \mathrm{E}\right)$. Grandparents were collected from the wild and mated artificially in the laboratory following standard in vitro fertilization procedure for sticklebacks (Divino and Shultz, 2014). F1-offspring were reared in 1.4-1 tanks in an Allentown Zebrafish Rack Systems (Aquaneering Inc., San Diego, USA). Water temperature was maintained at $17^{\circ} \mathrm{C}\left( \pm 0.5^{\circ} \mathrm{C}\right)$ and partial water changes were made regularly by replacing water in the reservoir tank connected to each rack. A 14:10 Light:Dark rhythm was maintained throughout the experiments. Following absorption of the yolk sac, fry was fed daily with Artemia nauplii and subsequently with a mixture of live Artemia nauplii and frozen Cyclops 29-56 days post-hatching (dph), with frozen Cyclops and chironomid larvae at 57-84 dph, and with frozen chironomid larvae thereafter. The feedings occurred twice a day, and the food was provided ad libitum. Once the $\mathrm{F}_{1}$-offspring were sexually mature, one male and one female from each cross were randomly chosen and allowed to mate to produce $\mathrm{F}_{2}$ offspring. For the Helsinki $\mathrm{x}$ Rytilampi cross (HEL x RYT), $F_{1}$ parents produced seven successive clutches yielding a total of $287 \mathrm{~F}_{2}$-offspring in September and October 2008. This mating procedure was followed at different dates for the Helsinki $\mathrm{x}$ Pyöreälampi (HEL x PYO; mating: July-Sep 2012, F 2 breeding: July 2012-Apr 2013) and the Helsinki $x$ Bynästjärnen (HEL x BYN; mating: Nov 2013- Jan 2014, $F_{2}$ breeding: Nov 2013-Aug 2014) crosses which respectively produced 288 and $313 \mathrm{~F}_{2}$-offspring. The $\mathrm{F}_{2}$ clutches were reared in $1.4 \mathrm{~L}$ tanks in an Allentown Zebrafish rack (Aquaneering Inc., San Diego, CA) until six days post-hatching (dph). After that, the fish were transferred to four Allentown Zebrafish racks for individual rearing and fed with live Artemia nauplii. Individual fish were housed in separate $1.4 \mathrm{~L}$ tanks and reared in the same conditions described above for their parents.

For the Helsinki cross, a total of 178 adult fish were sampled from a marine population from the Baltic Sea in Helsinki $\left(60^{\circ} 13^{\prime} \mathrm{N}, 25^{\circ} 11^{\prime} \mathrm{E}\right)$ in April 2016 and 
brought to the University of Helsinki aquarium facility for artificial mating. Full-sib/Half-sib $\mathrm{F}_{1}$ families were generated using the same standard mating procedure for sticklebacks described above by mating one female to two different males. Once the eggs had hatched and larvae started feeding, the families were thinned to 25 offspring per family and moved to two large aquaria so that about half of each family were placed in each unit. The larvae were mass-reared in these aquaria, and their family identity was later identified from the genotype data (see below). After nine months, the experiment was terminated and a total of $1069 \mathrm{~F}_{1}$ offspring distributed across 87 families were used for this study.

\section{Phenotypic data}

We obtained phenotypic data for all individuals of the four datasets on three morphological traits: body length, body depth and length of the pelvic spines, known to have heritable basis (Shimada et al., 2011; Kemppainen et al., 2020) and be involved in adaptive habitat differentiation between freshwater and marine ninespined sticklebacks (Karhunen et al., 2014). All individuals were photographed next to a millimeter scale with a digital camera, and images were used to measure body length and body depth using the tpsDig software (v.2.10; Rohlf, 2006). Body length was measured as the distance between the tip of the snout and the base of the posterior end of the hypural plate. Body depth corresponded to the distance between landmarks 3 and 12 in Herczeg et al., (2010). Length of the pelvic spines were measured using digital calipers to the nearest $0.01 \mathrm{~mm}$. We did not focus on the asymmetry of pelvic spines (but see: Blouw \& Boyd, 1992; Bell et al., 2007; Coyle et al., 2007) but rather decided to use the mean values of the left- and rightside measurements as the focal trait. All measurements were made by the same person twice, and the repeatabilities were $>0.9(p<0.001)$ for all traits (see also Yang et al., 2016, Kemppainen et al., 2021). Sex information for all samples was obtained first by genotyping all individuals at a sex-specific microsatellite locus (Stn 19; Shikano et al., 2011), and subsequently confirmed with sex-specific SNPs. After correcting for missing values, the final datasets contained full phenotypic record for 278 (HEL x RYT cross), 279 (HEL x PYO cross) and 301 (HEL x BYN cross) $F_{2}$ individuals and $936 F_{1}$ individuals (Helsinki crosses).

\section{SNP genotyping}

For the $\mathrm{F}_{2}$ crosses, restriction-site associated DNA sequencing approach (RAD; Davey et al., 2011) was used to obtain a large panel of single nucleotide polymorphisms as earlier described in Rastas et al. (2015). Two of the crosses used here HEL x RYT and HEL x PYO correspond to their "HR" and "HP" crosses, respectively, and the same methodology was applied to our third HEL x BYN cross. Briefly, DNA from alcohol-preserved fin clips was extracted using phenolchloroform method (Taggart et al. 1992) for library constructions and fragmented to 300-500bp fragments using the Pst1 restriction enzyme. DNA fragments were gel purified and subsequently ligated to adaptors and library-specific barcodes, after which the libraries were pooled. The pooled libraries were sequenced using 45bp single-end strategy on the Illumina HighSeq 2000 platform at BGI Genomics (Hong-Kong Co. Ltd.). Raw reads were processed by removing adapters and barcodes and the read quality was checked with FastQC (Andrews, 2010). Raw genotype data consisted of 21 832, 22603 and 21747 SNPs for the HEL x RYT, HEL $x$ PYO and HEL x BYN, respectively.

The Helsinki crosses SNP data was the same as in Kivikoski et al. (2020a). In summary, 50568 SNPs were obtained from sequencing/genotyping by Diversity Arrays Technology (Pty Ltd, Australia), using their DarTseq technology.

\section{Construction of the Genomic Relationship Matrices}

We estimated two types of matrices describing the relationship among individuals in each dataset: i) pedigree-based relationship matrices (PRM), corresponding to the theoretical expectation of sibling relatedness based on their pedigree and ii) SNP-based GRM corresponding to the estimated fraction of the genome shared IBD between siblings. The PRM were constructed from the pedigree structure describing each dataset using the nadiv R package (Wolak, 2012) which 
allowed us to compute both additive $\left(\mathrm{PRM}_{\mathrm{ADD}}\right)$ and dominance (PRM $\mathrm{POM}_{\text {) }}$ matrices using the makeA() and make $D$ () functions, respectively.

For the construction of the GRM, we used two methods. First, we used a modified construct of the additive GRM originally proposed by VanRaden (2008) as follows:

$$
G R M_{A D D}=\frac{W W^{\prime}}{\operatorname{trace}\left(W W^{\prime}\right) / n}
$$

where $W$ is the marker matrix of additive coefficients and $n$ the number of individuals, as implemented in the snpReady R package (Granato et al., 2018) for each dataset to construct GRMs from autosomal loci. This allowed us to produce for each dataset both additive and dominance variance matrices $\left(\mathrm{GRM}_{\mathrm{ADD}} \& \mathrm{GRM}_{\mathrm{DOM}}\right.$; Granato et al., 2018). Second, we also constructed additive and dominance genetic variance using the GCTA software (Yang et al., 2011) from autosomal loci for each dataset. Prior to using both approaches, we pruned the datasets from markers with a minimum allele frequency (MAF) lower than 0.01 and included only markers with a maximum of $10 \%$ missing data. This resulted in a total of 12679, 16869, 15216 and 15147 informative SNPs for the HEL $x$ RYT, HEL $x$ PYO, HEL $x$ BYN and Helsinki crosses datasets respectively.

\section{Estimation of variance components and heritabilities}

Three major quantitative genetic parameters underlying trait variability were estimated: additive genetic variance $\left(\mathrm{V}_{\mathrm{A}}\right)$, dominance variance $\left(\mathrm{V}_{\mathrm{D}}\right)$ and heritability $\left(h^{2}\right)$. We employed the 'animal model' approach (Kruuk, 2004), a random effect model using the PRM or GRM as the covariance of the random effect linking to the phenotypes, to estimate these parameters using the relatedness among full- and halfsibs in each dataset characterized in each relationship matrix. The Bayesian approach implemented in the MCMCglmm R package (Hadfield, 2010) was used to run animal models for each trait and each dataset separately by fitting sex as a fixed effect and implementing additive and dominance effect from either type of relationship matrix (PRM or GRM) as random effects. We ran each model for 503,000 Markov Chain Monte Carlo (MCMC) iterations with a burn-in period of 3,000 , and thinning every $100^{\text {th }}$ iteration, resulting in $5000 \mathrm{MCMC}$ posterior samples. Model convergence was checked visually from the mixing of MCMC chains by inspecting trace plots of many different model parameters and using the Heidelberger and Welch convergence test (Hadfield 2010). We calculated heritability as the ratio of $V_{A}$ to total phenotypic variance $\left(\mathrm{V}_{\mathrm{P}}\right)$ for a given trait. To provide further comparisons between estimates obtained with the two types of GRM we also estimated all variance components and heritabilities using the frequentist approach implemented in GCTA (Yang et al., 2011) by running Genome-based Restricted Maximum-Likelihood (GREML) models. Finally, meaningful comparisons of variance components estimated using different relationship matrices (here, GRM \& PRM) must refer to a common reference population wherein genetic variance is estimated (Speed \& Balding 2015, Legarra 2016). Specifically, models using PRM will estimate variance in the base population of the pedigree (i.e. the unrelated founders) while models using GRM will do so within the set of genotyped individuals (Legarra 2016; Joshi et al. 2020). Here, we followed the approach of Legarra (2016) and adjusted all variance components as follows:

$$
\sigma_{a d j}^{2}=\sigma^{2} \cdot(\overline{\operatorname{diag}(K)}-\bar{K})=\sigma^{2} \cdot D_{k}
$$

where $\sigma^{2}$ is the variance component estimated from the animal model and $K$ the corresponding relationship matrix (Legarra 2016; Joshi et al., 2020). By doing so, we set the reference population as the full-siblings samples and provide meaningful comparisons between the PRM and GRM-based models. Moreover, this allowed to account for inbreeding in our $\mathrm{F}_{2}$ crosses. Indeed, as our $\mathrm{F}_{2}$ crosses data consisted exclusively of full-sibs whose parents are also full-sibs, the PRM of the $F_{2}$ has 1 in the diagonal (i.e. self-relationship), while off-diagonal elements are 0.75 due to the full-sib mating of the $F_{1}$ parents. Hence, variance components in the $\mathrm{F}_{2}$ crosses must be corrected with $D_{k}=1-0.75=$ 0.25 . 


\section{Chromosome partitioning of the genetic variance}

To investigate the polygenic bases of the three morphological traits, we estimated the proportion of phenotypic variance explained by SNPs from each separate chromosome. To this end, we used the Genomic Best Linear Unbiased Prediction (G-BLUP) approach from GCTA. We calculated a GRM for each chromosome and a GRM for all but the focal chromosome and estimated genetic variance parameters with the GREML approach implemented in GCTA. Models were run independently for each trait and each chromosome for all crosses.

\section{Results}

\section{Distribution of IBD-sharing values}

The GRM estimated from the Helsinki cross data was well representative of the full-sib/half-sib structure of the data (Fig. 1) with a high frequency of full-siblings sharing half of their genome IBD (0.5; Fig. 1$)$ and halfsiblings showing an average relatedness coefficient of 0.25 . Most founders were 'unrelated' (IBD sharing < 0.01 ), but we did find some degree of relatedness among parents despite the random nature of the sampling (Fig. 1).

The relatedness among full-sibs from each of the $F_{2}$ intercross family estimated with the modified VanRaden method (VanRaden 2008) was not as straightforward to interpret (Fig. S1). In all crosses, the mean relatedness coefficient was centered around zero with pairs of individuals showing either positive or negative relatedness. Results obtained from the GCTA software were highly similar to those obtained with snpReady (Fig. S1). In order to get more meaningful (i.e., interpretable on a $[0-1]$ scale) values of relatedness in the $F_{2}$ crosses, we re-estimated IBD proportions using the KING software (Manichaikul et al., 2010). Relationship inference from KING was more representative of the pedigree structure and the mean relatedness for all three $F_{2}$ crosses were similar and slightly under the 0.5 theoretical expectation for fullsibs: 0.462 (HEL x RYT), 0.465 (HEL x PYO) and 0.456 (HEL x BYN; Fig. 2).

\section{Quantitative genetics parameters}

In all datasets, all variance components obtained with MCMCglmm using GRM were estimated more accurately (as represented by the narrower 95\% Highest Posterior Density [HPD] intervals) compared to PRM (Table 1).

In the Helsinki cross, total phenotypic variance $\left(\mathrm{V}_{\mathrm{P}}\right)$ estimates were very similar regardless of the type of GRM used and consistently higher for standard length (SL) compared with pelvic length (PL) and body depth (BD), respectively (Table 1). Additive and dominance genetic variance were estimated more accurately using the GRM than PRM in the Helsinki cross, and the estimates were higher for SL compared to PL and BD, respectively (Table 1). Overall, the contribution of dominance genetic variance $\left(V_{D}\right)$ in all traits for these dataset were small (Table 1). Heritability estimates using GRM were very low (PL) to moderate (SL), and consistently more accurate compared with estimates using PRM (Table 1). Interestingly, when removing the PRM $_{\text {DOM }}$ from the animal model using the Helsinki cross, estimates of variance components were very similar to those obtained using GRM (Table S1). However, variance components estimated with GCTA were higher for the Helsinki cross than those obtained with MCMCglmm (Table S2).

In the $\mathrm{F}_{2}$ intercrosses, models using PRM were unable to estimate meaningful values of $h^{2}$ as all 95\% HPD intervals spanned between $<0.01$ and $>0.9$. Based on the more accurate GRM models, we found the highest heritability value for pelvic spine length in the HEL $x$ RYT cross (0.740 [0.582; 0.824]; Table 1$)$ and the lowest also for pelvic spine length in the HEL x PYO cross (Table 1). We also found low contribution of dominance genetic variance to the total phenotypic variance in all $F_{2}$ crosses (Table 1). Estimates of variance components based on the $\mathrm{GRM}_{\mathrm{ADD}}$ and GRM $_{\text {DOM }}$ using the GCTA software were in agreement with those estimated with $M C M C g \operatorname{lmm}$ for all traits and all crosses (Table S2).

\section{Chromosome partitioning}


Overall, the proportion of variance explained by each individual chromosome for each trait was low to moderate in all crosses (Fig. 3,4), except for pelvic spine length (PL) in the HEL x RYT cross (Fig. 4) where Chromosome 7 explained 66\% (SE 0.086) of the total phenotypic variance. In the Helsinki crosses, we found significant regional heritability for body depth and pelvic length in linkage groups 6 and 5, respectively (Fig. 3). In the $F_{2}$ crosses, different chromosomes contributed to the total phenotypic variance of all traits between the three datasets (Fig. 4).

\section{Discussion}

The results show that genome-wide IBD information derived from SNP markers using even a single large full-sib family can yield meaningful estimates of quantitative genetic parameters for ecologically important traits, and greatly improve their accuracy as compared to traditional pedigree based estimates. Moreover, implementation of the GRM in the animal model allowed for greater model complexity, particularly by adding random effect term for the estimation of dominance genetic variance. In line with results of earlier studies, which have estimated dominance variance components for morphological traits (Roff \& Emerson 2007, reviewed in Wolak \& Keller 2014), the dominance contributions for all traits were negligible in our data. In the following, we discuss the usefulness and limitations inherent to the full-sib approach, and its potential utility for evolutionary studies of wild populations.

\section{Estimation of genome-wide IBD from SNP markers}

By definition, relatedness estimation from genomewide IBD is used to describe the actual proportion of genome sequence shared between any two individuals. Whereas whole-genome sequencing (WGS) is increasingly available to population geneticists, RAD sequencing remains the tool of the trade in the field. Hence, estimation of IBD sharing from scattered markers (as used in the present study) rather than whole genome sequences could yield biased relatedness estimates and consequently, biased heritability estimates (Gienapp et al 2017). However, available results from quantitative genomic studies from the wild suggest that unbiased estimates of heritability can be obtained with as little as < 20.000 SNPs (Bérénos et al., 2014; Gienapp et al., 2017). Heritability estimates consistent with pedigree-based estimates were also obtained from wild populations of the blue tits (Cyanistes caeruleus ogliastrae) using less than 500 individuals genotyped at 50.000 autosomal SNPs (Perrier et al., 2018). Our four datasets fall within this range, both in terms of number of individuals and markers used and should thus provide reasonable estimates of relatedness coefficients and associated variance components.

High variance in IBD sharing among full-siblings is expected to yield more accurate estimates of variance components and heritability than pedigree-based relationships (Visscher et al. 2006). In humans, relatedness among full-sibs can vary substantially around the 0.50 expectation: Visscher et al. (2006) reported IBD sharing among full-sibs to range from 0.317 to 0.617 . Here, taking the Helsinki crosses dataset as representative of a wild outbred population, we found that the average IBD sharing to span much wider range than in humans (mean IBD sharing: 0.505, min: 0.239; max: 0.754). In other words: some full-sib pairs did not share more of their genome in common than half-siblings, while others shared more than expected from brother-sister mating. Variance in IBD sharing between siblings is inherently linked to the recombination process and the number of crossovers occurring during meiosis: higher number of crossovers will lead to lower variance in IBD (Risch \& Lange, 1979; Kivikoski et al., 2020b). Linkage maps constructed from human data are on average twice the length as that from P. pungitius (Broman et al., 1998; Kivikoski et al., 2020a) indicating more crossovers in the human genome than in that of the nine-spined stickleback. Therefore, it is possible that this difference in the recombination landscape between the two species is responsible for the different range of IBD proportions among siblings. Regardless of the mechanism behind the higher variance in the nine-spined stickleback IBD, our results confirm that this species, and likely also its 
close relative, the three-spined stickleback (Gasterosteus aculeatus), sharing similar genetic architecture and cross-over pattern (Kivikoski et al., 2020b), can be particularly suitable models for quantitative genomic studies of wild populations (Merilä, 2013).

\section{Implementation of GRM in the animal model}

A central result of our study is that integrating the GRM constructed from SNP data in the animal model greatly improves the accuracy (and thereby possibly also precision) of heritability and variance components estimates compared to pedigree-based relationship matrix. This is a particularly important result for evolutionary biologists, as accuracy (and precision) of variance component estimates influence predicted responses to selection and phenotypic evolution in the wild (Lynch \& Walsh, 1998; Charmantier et al., 2014; Walsh \& Lynch, 2018). In practice, implementation of the GRM in the animal model is rather straightforward: one simply needs to append the $n \times n$ matrix (where $n$ is the number of individuals) of relatedness coefficient obtained from any software capable of IBD computation from genome-wide SNP data (e.g., PLINK, Purcell et al., 2007; GCTA, Yang et al., 2010; KING, Manichaikul et al., 2010). By using the Bayesian MCMCglmm R package (Hadfield, 2010) to conduct animal model analyses, we wanted to assess the potential limitations of this widely used method to handle different types of GRM. We found that MCMCglmm was sensitive to non-positive definite matrices and was unable to run the animal model when using GRM estimated from other widely used software packages (e.g., PLINK, KING). Although this issue can be inherent to the datasets used here, it is likely that similar issues can be encountered by other users. Hence, further studies focused on the development of animal model approaches to accommodate for different types of GRMs (see e.g., Zhao et al.,, 2018) is warranted.

The results further demonstrate that use of the GRM allowed for greater model complexity by implementation of the dominance matrix $\left(\mathrm{GRM}_{\mathrm{DOM}}\right)$ as an additional random term to estimate $\mathrm{V}_{\mathrm{D}}$. Until to date, dominance genetic variance has been often ignored in the evolutionary genetic literature (Wolak \& Keller, 2014). This is in part due to the fact that founding theoretical work predicted that non-additive genetic effects should contribute little on quantitative trait variance (Fisher, 1958), a prediction that has been later verified in some empirical studies (e.g., Hill et al., 2008; Zhu et al., 2015; Class \& Brommer, 2020; but see: Merilä et al. 2004). Nevertheless, a body of theoretical and empirical work suggest that dominance variance can indeed account for a significant proportion of phenotypic variance in the wild when populations are finite and genetically structured (e.g., Wright, 1931; Crnokrak \& Roff, 1995; Kosova et al., 2010). Hence, the importance (or lack thereof) of dominance genetic variance cannot be taken for granted and requires empirical data. Two results of particular importance regarding dominance variance from our study are worth high lighting. First, we found that dominance genetic variance accounted for a very low proportion of the total phenotypic variance for all traits in all datasets. On average, it accounted for $0.19 \%$ of $\mathrm{V}_{\mathrm{P}}$ across datasets compared to $\mathrm{V}_{\mathrm{A}}$, which explained on average $21.2 \%$ of $\mathrm{V}_{\mathrm{P}}$. Thus, our results are in line with the expectation that dominance variance in morphological traits is negligible. Second, the results further demonstrate the difficulty in obtaining accurate estimates of $V_{D}$ from family data. Here, even when using an appropriate crossing design (i.e., maternal half-sib; Wolak \& Keller, 2014) with relatively large number of families and individuals, we found that estimates of $V_{D}$ obtained from pedigree-based analyses were overestimated as compared to the ones obtained with GRM. As a result, accuracy of estimation of the other components of variance in animal model was affected, and heritability estimates became biased downwards. Hence, the results advocate the use of the GRM for animal models aiming at partitioning additive and dominance variance components, regardless of the sample size and crossing design. It is also perceivable that studies with smaller datasets (ca. hundreds of individuals) should greatly benefit from the use of GRM as previously noted by Perrier et al., (2018). 
Genetic architecture of morphological traits in $P$. pungitius

The pace of adaptive evolution is determined by the amount of heritable variation in traits under selection, but the heritability of the given trait may be specific to given population, habitat, or sex (Price \& Schluter, 1991; Roff, 1997; Hoffmann \& Merilä, 1999; Wilson et al., 2010). Here we studied three morphological traits previously shown to be involved in the phenotypic differentiation between different ecotypes of $P$. pungitius. Following the colonization of lakes and ponds from the marine environment, freshwater populations of $P$. pungitius have repeatedly evolved distinct morphologies such as increased body size (i.e., gigantism; Herczeg et al. 2009), changes in body shape (Herczeg et al. 2010) and pelvic reduction (Kemppainen et al. 2021). A reasonable assumption is therefore that each of these traits displayed sufficient heritability in the marine ancestral population to respond to the selection pressures associated with the colonization of the freshwater habitat. In the current study, the Helsinki population may be considered as representative - i.e., genetically and morphologically similar (Shikano et al., 2010, Karhunen et al., 2014) of an ancestral marine population of $P$. pungitius, and, in line with the previous assumption, we found significant heritability for body size and body depth in this population. Further dissection of the genetic architecture of body size and depth revealed that heritability of both traits was partitioned across the genome, with several chromosomes cumulatively accounting for small proportions of total phenotypic variance. Thus, these results further confirm the polygenic nature of body size in $P$. pungitius (Laine $e t$ al., 2013) and provide insight into the genetic architecture of body depth in this species. Thus, body size and depth appear to be 'classical' quantitative traits influenced by many genes of small effects (Visscher $e t$ al. 2007) rather than by few large effect loci. However, our analysis of pelvic spine length in the Helsinki crosses revealed a different picture: we found little phenotypic and additive genetic variance underlying this trait, and thus, very low heritability $\left(h^{2}<0.01\right)$ of pelvic spine in this population. Regressive evolution of the pelvic apparatus (hereafter pelvic reduction) has been much studied in stickleback fishes (Bell et al., 1993; Gibson, 2005; Chan et al., 2010; Xie et al., 2019; Kemppainen et al., 2021). Kemppainen et al., (2021) showed that pelvic reduction is a heterogeneous process in P. pungitius, and that the heritability of this trait varies from population to other. Therefore, it is possible that the alleles (or combination of alleles) underlying pelvic variation are not segregating in our study population, thus explaining the low heritability of this trait. Alternatively, it is possible that the pelvic phenotype in this population is close to an adaptive optimum where stabilizing selection has depleted most of the additive genetic variance underlying this trait (Fisher, 1958; Karavolias et al.,, 2020). Additional studies of selection intensity and in-depth analyses of the genetic architecture of pelvic spines (e.g. GWAS) may shed more light on the genetic bases of this trait in the Baltic marine populations of P. pungitius.

In the $F_{2}$ crosses, our animal model approach also revealed heterogeneity in the quantitative genetic parameters underlying body size and depth, as well as the pelvic spine. Despite the differences in the point estimates (i.e., modes of the posterior distribution), we did not find significant differences in heritability of body size and body depth between the three crosses. However, we found strong differences in heritability of the pelvic spine length, with all three crosses displaying significantly different heritabilities to one another. This result is very much in line with those from Kemppainen et al., (2021), where the proportions of variance (PVE) explained by all SNPs are highly similar to our estimates of heritability of pelvic length for each of the crosses (see Table 2 in Kemppainen et al., 2021). Interestingly, our chromosome partitioning analyses revealed heterogeneous chromosome contributions to the overall phenotypic variance for all three morphological traits, between all crosses. Differences in the genetic architecture of the same trait between these crosses indicate that different alleles govern phenotypic variation in the three different pond populations despite their adaptation to the same environment. As recently demonstrated by 
Kemppainen et al. (2021) and Fang et al., (2021), marine populations of $P$. pungitius display relatively high level of genetic isolation-by-distance resulting in higher population structure in the sea. Consequently, and conversely to the classical model of parallel evolution described in the three-spined stickleback (Gasterosteus aculeatus; Colosimo et al., 2005), repeated colonization of similar freshwater habitats by $P$. pungitius was likely initiated by populations with different allele frequencies at causal loci underlying traits under selection. The likelihood of parallel evolution is therefore expected to be low among freshwater populations of $P$. pungitius, as was demonstrated for pelvic phenotypes by Kemppainen $e t$ al., (2021). However, whether the same processes underlie the differences in genetic architecture of body size and depth observed in this study require further investigations. Nevertheless, the similarity between the results of our chromosome partitioning analyses of pelvic spine length and the from Kemppainen et al. (2021) obtained with state-of-the-art QTL-mapping approach (Li et al., 2017, 2018) suggest that our results provide a reasonable approximation of the genetic architecture of quantitative traits. Further analyses of these datasets using similar QTL-mapping approaches as used in Kemppainen et al., (2021) may reveal comparable patterns of heterogeneous architecture in body size and depth.

\section{Methodological and interpretational issues}

The full-sib families used in this study were reared in laboratory rather than in the wild, and hence, the relevance of the estimated quantitative genetic parameters for evolutionary inference can be debated as heritabilites and variance components can change if the environmental conditions change (Hoffmann \& Merilä 1999, Charmantier \& Garant 2005). Since beneficial and homogenous environmental conditions, such as implemented in this study, can be associated both with high and low heritabilities, it is difficult to know whether and if our estimates under- or overestimate corresponding estimates in the wild. Similarly, since the used $\mathrm{F}_{2}$-crosses constitute hybrid populations and that genetic introgression increases genetic diversity, the estimates of genetic variance derived from these crosses may represent artificially high values. Nevertheless, the fact that estimates from different $F_{2}$ and Helsinki crosses were all fairly similar suggest that the latter effect was probably of not any greater concern. While further studies based on sampling of relatives from wild are warranted to address the problem of environmental effects on estimated parameters, our findings can be viewed as "proof-of-principle" showing that accurate estimates of quantitative genetic parameters can obtained with relatively low sampling effort of single full-sib families.

\section{Conclusions and future prospects}

In this paper, we have explored the utility of full-sib data in obtaining estimates of quantitative genetic parameters for non-model organisms and demonstrated that approach works but poses also some challenges. The main logistic challenge involved with the study of genetics of ecologically important traits in wild populations is in obtaining the pedigree data required for quantitative genetic inference. The approach adopted in this study to estimate quantitative genetic parameters from a data derived from a single (or handful of) full-sib family (families) should be transferable to the studies of variety of wild organisms. While the applicability of this approach will be limited for organisms with small propagules sizes, it could be readily used for instance in many plant, insect and fish systems to obtain in situ estimates of quantitative genetic parameters in different populations in their natural environments. However, since the accuracy genomic heritability method depends on the variance of genome-wide IBD (Xu, 2006; Visscher, 2009), the genomic characteristics of the model system will influence the utility and required sampling design in obtaining parameter estimates of sufficient accuracy.

\section{Acknowledgements}

We thank Federico Calboli and Ari Löytynoja for constructive comments on the earlier version of the manuscript. We thank Gabor Herczeg, Abigel Gonda, Yukinori Shimada, Mirva Turtiainen, Chris Eberlein, Takahito Shikano, Laura Hänninen, Kirsi Kähkönen, 
Miinastiina Issakainen and Sami Karja for help in fish breeding and DNA extractions, and Jing Yang for help with fish phenotyping. We thank Mikko Kivikoski for helpful discussions for estimation of IBD proportions. The computing resource support from CSC - the Finnish IT Center for Science Ltd administered by the Ministry of Education and Culture, Finland is gratefully acknowledged. Our study was supported by the Academy of Finland (grant nos. 129662, 134728 and 218343 to J.M.).

\section{Ethical note}

All experimental protocols were approved by permission (ESLHSTSTH223A) form the National Animal Experiment Board, Finland.

\section{Data availability}

Raw sequence reads for the $F_{2}$ crosses have been submitted to NCBIs short-read archives with accession nos.: PRJNA673430 and PRJNA672863. Data for pelvic phenotypes and standard length are available on Dryad: https://doi.org/10.5061/dryad.76hdr7str.

\section{Author contributions}

J.M. Z.L M.S. P.R. and A.F. conceived the study; P.R. contributed to pre-processing and getting the genotype data; A.F. and Z.L. analyzed the data; J.M. and A.F. led the writing of the manuscript. All authors contributed critically to the drafts and gave final approval for publication.

\section{References}

Ab Ghani, N. I., Herczeg, G., \& Merilä, J. (2012). Body size divergence in nine-spined sticklebacks: disentangling additive genetic and maternal effects. Biological Journal of the Linnean Society, 107(3), 521-528.

Andrews S. (2010). FastQC: a quality control tool for high throughput sequence data. Available online at:

http:/www.bioinformatics.babraham.ac.uk/projec ts/fastqc

Bell, M. A., Khalef, V., \& Travis, M. P. (2007). Directional asymmetry of pelvic vestiges in threespine stickleback. Journal of Experimental Zoology Part B: Molecular and Developmental Evolution, 308(2), 189-199.

Bell, M. A., Ortí, G., Walker, J. A., \& Koenings, J. P. (1993). Evolution of pelvic reduction in threespine stickleback fish: a test of competing hypotheses. Evolution, 47(3), 906-914.

Bérénos, C., Ellis, P. A., Pilkington, J. G., \& Pemberton, J. M. (2014). Estimating quantitative genetic parameters in wild populations: a comparison of pedigree and genomic approaches. Molecular Ecology, 23(14), 3434-3451.

Blouw, D. M., \& Boyd, G. J. (1992). Inheritance of reduction, loss, and asymmetry of the pelvis in Pungitius pungitius (ninespine stickleback). Heredity, 68(1), 33-42.

Broman, K. W., Murray, J. C., Sheffield, V. C., White, R. L., \& Weber, J. L. (1998). Comprehensive human genetic maps: individual and sex-specific variation in recombination. American Journal of Human Genetics, 63(3), 861-869.

Bruneaux, M., Johnston, S. E., Herczeg, G., Merila, J., Primmer, C. R., \& Vasemagi, A. (2013).

Molecular evolutionary and population genomic analysis of the nine-spined stickleback using a modified restriction-site-associated DNA tag approach. Molecular Ecology, 22(3), 565-582.

Chan, Y. F., Marks, M. E., Jones, F. C., Villarreal, G., Shapiro, M. D., Brady, S. D., Southwick, A. M., Absher, D. M., Grimwood, J., \& Schmutz, J. (2010). Adaptive evolution of pelvic reduction in sticklebacks by recurrent deletion of a Pitx 1 enhancer. Science, 327(5963), 302-305.

Charmantier, A., \& Garant, D. (2005). Environmental quality and evolutionary potential: lessons from wild populations. Proceedings of the Royal Society B: Biological Sciences, 272(1571), 14151425.

Charmantier, A., Garant, D., \& Kruuk, L. E. B. (2014). Quantitative genetics in the wild. Oxford University Publishing.

Class, B., \& Brommer, J. E. (2020). Can dominance genetic variance be ignored in evolutionary quantitative genetic analyses of wild 
populations? Evolution, 74(7), 1540-1550.

Colosimo, P. F., Hosemann, K. E., Balabhadra, S., Villarreal, G., Dickson, M., Grimwood, J., Schmutz, J., Myers, R. M., Schluter, D., \& Kingsley, D. M. (2005). Widespread parallel evolution in sticklebacks by repeated fixation of ectodysplasin alleles. Science, 307(5717), 19281933.

Coyle, S. M., Huntingford, F. A., \& Peichel, C. L. (2007). Parallel evolution of Pitx1 underlies pelvic reduction in Scottish threespine stickleback (Gasterosteus aculeatus). Journal of Heredity, 98(6), 581-586.

Crnokrak, P., \& Roff, D. A. (1995). Dominance variance: associations with selection and fitness. Heredity, 75(5), 530-540.

De-la-Cruz, I. M., Merilä, J., Valverde, P. L., FloresOrtiz, C. M., \& Núñez-Farfán, J. (2020). Genomic and chemical evidence for local adaptation in resistance to different herbivores in Datura stramonium. Evolution, 74(12), 26292643.

Duntsch, L., Tomotani, B. M., de Villemereuil, P., Brekke, P., Lee, K. D., Ewen, J. G., \& Santure, A. W. (2020). Polygenic basis for adaptive morphological variation in a threatened Aotearoa| New Zealand bird, the hihi (Notiomystis cincta). Proceedings of the Royal Society B, 287(1933).

Edwards, D., \& Batley, J. (2010). Plant genome sequencing: applications for crop improvement. Plant biotechnology Journal, 8(1), 2-9.

Falconer, D. S., \& Mackay, T. F. C. (1996). Introduction into quantitative genetics. Essex: Prentice Hall.

Fisher, R. A. (1918). The correlation between relatives on the supposition of Mendelian inheritance.

Transactions of the Royal Society of Edinburgh, 52(2), 399-433.

Fisher, R. A. (1958). The Genetical Theory of Natural Selection. The Clarandon Press.

Gervais, L., Hewison, A. J., Morellet, N., Bernard, M., Merlet, J., Cargnelutti, B., et al. (2020).
Pedigree-free quantitative genetic approach provides evidence for heritability of movement tactics in wild roe deer. Journal of Evolutionary Biology, 33(5), 595-607.

Gibson, G. (2005). The synthesis and evolution of a supermodel. Science, 307(5717), 1890-1891.

Gienapp, P., Fior, S., Guillaume, F., Lasky, J. R., Sork, V. L., Csilléry, K. (2017). Genomic quantitative genetics to study evolution in the wild. Trends in Ecology \& Evolution, 32(12), 897-908.

Gienapp, P., Calus, M. P., Laine, V. N., \& Visser, M. E. (2019). Genomic selection on breeding time in a wild bird population. Evolution Letters, 3(2), 142-151.

Granato, I. S. C., Galli, G., de Oliveira Couto, E. G., Bandeira e Souza, M., Mendonça, L. F., \& Fritsche-Neto, R. (2018). snpReady: a tool to assist breeders in genomic analysis. Molecular Breeding, 38(8), 1-7.

Hadfield, J. D. (2010). MCMC methods for multiresponse generalized linear mixed models: the MCMCglmm R package. Journal of Statistical Software, 33(2), 1-22.

Hayes, B., \& Goddard, M. (2010). Genome-wide association and genomic selection in animal breeding. Genome, 53(11), 876-883.

Herczeg, G, Gonda, A., \& Merilä, J. (2009a).

Evolution of gigantism in nine-spined sticklebacks. Evolution, 63(12), 3190-3200.

Herczeg, G, Gonda, A., \& Merilä, J. (2009b). Predation mediated population divergence in complex behaviour of nine-spined stickleback (Pungitius pungitius). Journal of Evolutionary Biology, 22(3), 544-552.

Herczeg, Gábor, Gonda, A., Kuparinen, A., \& Merilä, J. (2012). Contrasting growth strategies of pond versus marine populations of nine-spined stickleback (Pungitius pungitius): a combined effect of predation and competition? Evolutionary Ecology, 26(1), 109-122.

Herczeg, Gábor, Turtiainen, M., \& Merilä, J. (2010). Morphological divergence of North-European nine-spined sticklebacks (Pungitius pungitius): 
signatures of parallel evolution. Biological

Journal of the Linnean Society, 101(2), 403-416.

Hill, W. G., Goddard, M. E., \& Visscher, P. M. (2008). Data and theory point to mainly additive genetic variance for complex traits. PLoS Genet, 4(2), e1000008.

Hoffmann, A. A., \& Merilä, J. (1999). Heritable variation and evolution under favourable and unfavourable conditions. Trends in Ecology \& Evolution, 14(3), 96-101.

Joshi, R., Meuwissen, T. H., Woolliams, J. A., \& Gjøen, H. M. (2020). Genomic dissection of maternal, additive and non-additive genetic effects for growth and carcass traits in Nile tilapia. Genetics Selection Evolution, 52(1), 1-13.

Karavolias, N. G., Greenberg, A. J., Barrero, L. S., Maron, L. G., Shi, Y., Monteverde, E., Piñeros, M. A., \& McCouch, S. R. (2020). Low additive genetic variation in a trait under selection in domesticated rice. G3: Genes, Genomes, Genetics, 10(7), 2435-2443.

Karhunen, M., Ovaskainen, O., Herczeg, G., \& Merilä, J. (2014). Bringing habitat information into statistical tests of local adaptation in quantitative traits: A case study of nine-spined sticklebacks. Evolution, 68(2), 559-568.

Kemppainen, P., Li, Z., Rastas, P., Löytynoja, A., Fang, B., Yang, J., Guo, B., Shikano, T., \& Merilä, J. (2021). Genetic population structure constrains local adaptation in sticklebacks. Molecular Ecology. Accepted Author Manuscript. https://doi.org/10.1111/mec.15808

Kemppainen, P., Li, Z., Rastas, P., Löytynoja, A., Fang, B., Yang, J., Guo, B., Shikano, T., \& Merilä, J. (2021). SNP and phenotype data for $F_{2}$ crosses of Pungitius pungitius. https://doi.org/10.5061/dryad.76hdr7str

Kivikoski, M., Rastas, P., Löytynoja, A., \& Merilä, J. (2020a). New gains with a slimmer genome - An automated approach to improve reference genome assemblies.

bioRxiv. https://doi.org/10.1101/2020.08.18.255 596

Kivikoski, M., Rastas, P., Löytynoja, A., \& Merilä, J. (2020b). Effects of crossover interference on genomic recombination landscape. bioRxiv. https://doi.org/10.1101/2020.12.14.422614

Kosova, G., Abney, M., \& Ober, C. (2010).

Heritability of reproductive fitness traits in a human population. Proceedings of the National Academy of Sciences USA, 107(suppl 1), 17721778 .

Kruuk, L. (2004). Estimating genetic parameters in natural populations using the 'animal model'. Philosophical Transactions of the Royal Society of London. Series B: Biological Sciences, 359(1446), 873-890.

Laine, V. N., Shikano, T., Herczeg, G., Vilkki, J., \& Merilä, J. (2013). Quantitative trait loci for growth and body size in the nine-spined stickleback Pungitius pungitius L. Molecular Ecology, 22(23), 5861-5876.

Legarra, A. (2016). Comparing estimates of genetic variance across different relationship models. Theoretical Population Biology, 107, 26-30.

Leinonen, T., McCairns, R. S., O'hara, R. B., \& Merilä, J. (2013). QST-FST comparisons: evolutionary and ecological insights from genomic heterogeneity. Nature Reviews Genetics, 14(3), 179-190.

Li, Z., Guo, B., Yang, J., Herczeg, G., Gonda, A., Balázs, G., Shikano, T., Calboli, F. C. F., \& Merilä, J. (2017). Deciphering the genomic architecture of the stickleback brain with a novel multilocus gene-mapping approach. Molecular Ecology, 26(6), 1557-1575.

Li, Z., Kemppainen, P., Rastas, P., \& Merilä, J. (2018). Linkage disequilibrium clustering-based approach for association mapping with tightly linked genomewide data. Molecular Ecology Resources, 18(4), 809-824.

Li, Z., Löytynoja, A., Fraimout, A., \& Merilä, J. (2019). Effects of marker type and filtering criteria on QST-FST comparisons. Royal Society Open Science, 6(11), 190666.

Lush, J. L. (1943). Animal Breeding Plans. Second Edition. Iowa State Press. 
Lynch, M, \& Walsh, B. (1998). Genetics and Analysis of Quantitative Traits. Sinauer.

Lynch, M, \& Ritland, K. (1999). Estimation of pairwise relatedness with molecular markers. Genetics, 152(4), 1753-1766.

Malécot, G. (1948). Les Mathématiques de l'hérédité. Masson.

Manichaikul, A., Mychaleckyj, J. C., Rich, S. S., Daly, K., Sale, M., \& Chen, W.-M. (2010). Robust relationship inference in genome-wide association studies. Bioinformatics, 26(22), 2867-2873.

Manolio, T. A., Collins, F. S., Cox, N. J., Goldstein, D. B., Hindorff, L. A., Hunter, D. J., McCarthy, M. I., Ramos, E. M., Cardon, L. R., \& Chakravarti, A. (2009). Finding the missing heritability of complex diseases. Nature, 461(7265), 747-753.

Merilä, J. (2013). Nine-spined stickleback (Pungitius pungitius): an emerging model for evolutionary biology research. Annals of the New York Academy of Sciences, 1289, 18-35.

Merilä, J., \& Sheldon, B. C. (1999). Genetic architecture of fitness and nonfitness traits: empirical patterns and development of ideas. Heredity, 83(2), 103-109.

Merilä, J., Söderman, F., O'hara, R., Räsänen, K., \& Laurila, A. (2004). Local adaptation and genetics of acid-stress tolerance in the moor frog, Rana arvalis. Conservation Genetics, 5(4), 513-527.

Mousseau, T. A., Ritland, K., \& Heath, D. D. (1998). A novel method for estimating heritability using molecular markers. Heredity, 80(2), 218-224.

Nishio, M. \& Satoh, M. (2014) Including dominance effects in the genomic BLUP method for genomic evaluation. PLoS One. 2014; 9(1): e85792.

Perrier, C., Delahaie, B., \& Charmantier, A. (2018). Heritability estimates from genomewide relatedness matrices in wild populations: Application to a passerine, using a small sample size. Molecular Ecology Resources, 18(4), 838853.

Powell, J. E., Visscher, P. M., \& Goddard, M. E.
(2010). Reconciling the analysis of IBD and IBS in complex trait studies. Nature Reviews Genetics, 11(11), 800-805.

Price, T., \& Schluter, D. (1991). On the low heritability of life-history traits. Evolution, 45(4), 853-861.

Risch, N., \& Lange, K. (1979). Application of a recombination model in calculating the variance of sib pair genetic identity. Annals of Human Genetics, 43(2), 177-186.

Ritland, K. (1996). A marker-based method for inferences about quantitative inheritance in natural populations. Evolution, 50(3), 10621073.

Ritland, K. (2000). Marker-inferred relatedness as a tool for detecting heritability in nature. Molecular Ecology, 9(9), 1195-1204.

Robinson, M. R., Santure, A. W., DeCauwer, I., Sheldon, B. C., \& Slate, J. (2013). Partitioning of genetic variation across the genome using multimarker methods in a wild bird population. Molecular Ecology, 22(15), 3963-3980.

Roff, D. A. (1997). Heritability. In Evolutionary quantitative genetics (pp. 24-72). Springer.

Roff, D. A., \& Emerson, K. (2006). Epistasis and dominance: evidence for differential effects in life-history versus morphological traits. Evolution, 60(10), 1981-1990.

Rohlf, F. J. (2006). tpsDig, version 2.10. http://Life. Bio. Sunysb. Edu/Morph/Index. Html.

Shikano, T, Shimada, Y., Herczeg, G., \& Merila, J. (2010). History vs. habitat type: explaining the genetic structure of European nine-spined stickleback (Pungitius pungitius) populations. Molecular Ecology, 19(6), 1147-1161.

Shikano, Takahito, Herczeg, G., \& Merilä, J. (2011). Molecular sexing and population genetic inference using a sex-linked microsatellite marker in the nine-spined stickleback (Pungitius pungitius). BMC Research Notes, 4(1), 1-6.

Shikano, Takahito, Laine, V. N., Herczeg, G., Vilkki, J., \& Merilä, J. (2013). Genetic architecture of parallel pelvic reduction in ninespine sticklebacks. G3: Genes, Genomes, Genetics, 
3(10), 1833-1842.

Shimada, Y., Shikano, T., Kuparinen, A., Gonda, A., Leinonen, T., \& Merilä, J. (2011). Quantitative genetics of body size and timing of maturation in two nine-spined stickleback (Pungitius pungitius) populations. PLoS One, 6(12), e28859.

Sillanpää, M. J. (2011). On statistical methods for estimating heritability in wild populations. Molecular Ecology, 20(7), 1324-1332.

Sinclair-Waters, M., Ødegård, J., Korsvoll, S. A., Moen, T., Lien, S., Primmer, C. R., \& Barson, N. J. (2020). Beyond large-effect loci: large-scale GWAS reveals a mixed large-effect and polygenic architecture for age at maturity of Atlantic salmon. Genetics Selection

Evolution, 52(1), 1-11.

Speed, D., \& Balding, D. J. (2015). Relatedness in the post-genomic era: is it still useful? Nature Reviews Genetics, 16(1), 33-44.

Teacher, A. G. F., Shikano, T., Karjalainen, M. E., \& Merila, J. (2011). Phylogeography and genetic structuring of European nine-spined sticklebacks (Pungitius pungitius)-Mitochondrial DNA Evidence. Plos One, 6(5).

Tenesa, A., \& Haley, C. S. (2013). The heritability of human disease: estimation, uses and abuses. Nature Reviews Genetics, 14(2), 139.

Thompson, E. A. (2013). Identity by descent: variation in meiosis, across genomes, and in populations. Genetics, 194(2), 301-326.

VanRaden, P.M. (2008). Efficient methods to compute genomic predictions. Journal of Dairy Science, 91(11): 4414-23.

Varadharajan, S., Rastas, P., Löytynoja, A., Matschiner, M., Calboli, F. C. F., Guo, B., Nederbragt, A. J., Jakobsen, K. S., \& Merilä, J. (2019). Genome sequencing of the nine-spined stickleback (Pungitius pungitius) provides insights into chromosome evolution. bioRxiv. https://doi.org/10.1101/741751

Visscher, P. M. (2009). Whole genome approaches to quantitative genetics. Genetica, 136(2), 351.

Visscher, P. M., Macgregor, S., Benyamin, B., Zhu,
G., Gordon, S., Medland, S., Hill, W. G., Hottenga, J.-J., Willemsen, G., \& Boomsma, D. I. (2007). Genome partitioning of genetic variation for height from 11,214 sibling pairs. The American Journal of Human Genetics, 81(5), 1104-1110.

Visscher, P. M., Medland, S. E., Ferreira, M. A. R., Morley, K. I., Zhu, G., Cornes, B. K., Montgomery, G. W., \& Martin, N. G. (2006). Assumption-free estimation of heritability from genome-wide identity-by-descent sharing between full siblings. PLoS Genetics, 2(3), e41.

Walsh, B., \& Lynch, M. (2018). Evolution and Selection of Quantitative Traits. Oxford University Press.

Wilson, A. J., Reale, D., Clements, M. N., Morrissey, M. M., Postma, E., Walling, C. A., Kruuk, L. E. B., \& Nussey, D. H. (2010). An ecologist's guide to the animal model. Journal of Animal Ecology, 79(1), 13-26.

Wolak, M. E. (2012). nadiv: an R package to create relatedness matrices for estimating non-additive genetic variances in animal models. Methods in Ecology and Evolution, 3(5), 792-796.

Wolak, M. E., \& Keller, L. F. (2014). Dominance genetic variance and inbreeding in natural populations. Quantitative Genetics in the Wild, 104-127.

Wootton, R. J. (1976). Biology of the Sticklebacks. Academic Press.

Wright, S. (1931). Evolution in Mendelian populations. Genetics, 16(2), 97.

Xie, K. T., Wang, G., Thompson, A. C., Wucherpfennig, J. I., Reimchen, T. E., MacColl, A. D. C., Schluter, D., Bell, M. A., Vasquez, K. M., \& Kingsley, D. M. (2019). DNA fragility in the parallel evolution of pelvic reduction in stickleback fish. Science, 363(6422), 81-84.

$\mathrm{Xu}, \mathrm{S}$. (2006). Population genetics: separating nurture from nature in estimating heritability. Heredity, 97, 256-257.

Yang, J., Lee, S. H., Goddard, M. E., \& Visscher, P. M. (2011). GCTA: a tool for genome-wide complex trait analysis. American Journal of 
Human Genetics, 88(1), 76-82.

Zhao, J. H., Luan, J., \& Congdon, P. (2018). Bayesian linear mixed models with polygenic effects.

Journal of Statistical Software, 85(6), 1-27

Zhu, Z., Bakshi, A., Vinkhuyzen, A. A. E., Hemani, G., Lee, S. H., Nolte, I. M., van Vliet-

Ostaptchouk, J. V, Snieder, H., Esko, T., \&

Milani, L. (2015). Dominance genetic variation

contributes little to the missing heritability for

human complex traits. American Journal of

Human Genetics, 96(3), 377-385. 
bioRxiv preprint doi: https://doi.org/10.1101/2021.03.01.432833; this version posted March 1, 2021. The copyright holder for this preprint (which was not certified by peer review) is the author/funder, who has granted bioRxiv a license to display the preprint in perpetuity. It is made available under aCC-BY-NC-ND 4.0 International license.

\begin{tabular}{|c|c|c|c|c|c|c|c|c|c|}
\hline & & PRM & & & & GRM & & & \\
\hline Cross & Trait & $\mathbf{V}_{\mathbf{P}}$ & $\mathbf{V}_{\mathrm{A}}$ & $\mathbf{V}_{\mathbf{D}}$ & $\mathbf{h}^{2}$ & $\mathbf{V}_{\mathbf{P}}$ & $\mathbf{V}_{\mathrm{A}}$ & $\mathbf{V}_{\mathbf{D}}$ & $\mathbf{h}^{2}$ \\
\hline \multirow[t]{6}{*}{ Helsinki } & SL & 15.511 & $0.010[3.8 \mathrm{e}-$ & $15.250[6 \mathrm{e}-$ & $0.001[2.5 \mathrm{e}-$ & 15.291 & 3.849 & $0.005[2.9 \mathrm{e}-$ & 0.253 \\
\hline & & {$[13.826 ; 17.137]$} & $4 ; 3.743]$ & $4 ; 15.796]$ & $5 ; 0.242]$ & {$[13.631 ; 16.959]$} & {$[1.763 ; 5.849]$} & $4 ; 0.834]$ & {$[0.131 ; 0.381]$} \\
\hline & BD & 0.588 & $0.003[2 \mathrm{e}-$ & $0.003[2.7 \mathrm{e}-$ & $0.005[3.3 \mathrm{e}-$ & 0.593 & 0.129 & $0.001[1.7 \mathrm{e}-$ & 0.188 \\
\hline & & {$[0.521 ; 0.655]$} & $4 ; 0.249]$ & $4 ; 0.290]$ & $4 ; 0.412]$ & {$[0.522 ; 0.663]$} & {$[0.014 ; 0.226]$} & $4 ; 0.087]$ & {$[0.039 ; 0.384]$} \\
\hline & PL & 1.767 & $0.002[2 \mathrm{e}-$ & $0.002[2.7 \mathrm{e}-$ & $0.001[1.2 \mathrm{e}-$ & 1.778 & $0.002[1.7 \mathrm{e}-$ & $0.002[2.9 \mathrm{e}-$ & $0.001[8.8 \mathrm{e}-$ \\
\hline & & {$[1.621 ; 1.930]$} & $4 ; 0.147]$ & $4 ; 0.245]$ & $4 ; 0.082]$ & {$[1.623 ; 1.950]$} & $4 ; 0.096]$ & $4 ; 0.269]$ & $5 ; 0.053]$ \\
\hline \multirow{6}{*}{$\begin{array}{l}\text { HEL } x \\
\text { RYT }\end{array}$} & SL & $16.923[8.066$ & $0.013[3.9 \mathrm{e}-$ & $0.029[1.1 \mathrm{e}-$ & $1.4 \mathrm{e}-3[2.6 \mathrm{e}-$ & 17.259 & 4.819 & $0.002[2.2 \mathrm{e}-$ & 0.345 \\
\hline & & 19.881] & $5 ; 9.196]$ & $4 ; 18.44]$ & $6 ; 0.99]$ & {$[14.745 ; 21.970]$} & {$[2.232 ; 10.036]$} & $4 ; 0.812]$ & {$[0.145 ; 0.492]$} \\
\hline & $\mathrm{BD}$ & 0.349 & $0.001[6.8 \mathrm{e}-$ & $0.002[1 \mathrm{e}-$ & $0.004[1.5 \mathrm{e}-$ & 0.339 & 0.055 & $0.001[2.2 \mathrm{e}-$ & 0.164 \\
\hline & & {$[0.172 ; 0.403]$} & $5 ; 0.176]$ & $4 ; 0.359]$ & $4 ; 0.928]$ & {$[0.290 ; 0.417]$} & {$[0.015 ; 0.126]$} & $4 ; 0.038]$ & {$[0.061 ; 0.342]$} \\
\hline & PL & 2.097 & $0.003[6.7 \mathrm{e}-$ & $0.005[1.1 \mathrm{e}-$ & $0.003[2.46 \mathrm{e}-$ & 2.654 & 1.797 & $0.003[2.5 \mathrm{e}-$ & $0.740[0$. \\
\hline & & {$[1.028 ; 2.506]$} & $5 ; 1.131]$ & $4 ; 2.302]$ & $5 ; 0.975]$ & {$[2.076 ; 3.313]$} & {$[1.251 ; 2.645]$} & $4 ; 0.465]$ & $582 ; 0.824]$ \\
\hline \multirow{5}{*}{$\begin{array}{l}\text { HEL } x \\
\text { PYO }\end{array}$} & SL & 13.135 & $0.009[4.1 \mathrm{e}-$ & $0.042[1.1 \mathrm{e}-$ & $0.002[3.1 \mathrm{e}-$ & 13.097 & 2.682 & $0.002[1.5 \mathrm{e}-$ & 0.20 \\
\hline & & {$[6.110 ; 15.424]$} & $5 ; 7.063]$ & $4 ; 14.290]$ & $6 ; 0.991]$ & [11.160;16.349] & {$[0.854 ; 5.899]$} & $4 ; 0.742]$ & {$[0.076 ; 0.392]$} \\
\hline & $\mathrm{BD}$ & 0.742 & $0.001[4 \mathrm{e}-$ & $0.003[7.6 \mathrm{e}-$ & $0.004[5.5 \mathrm{e}-$ & 0.793 & 0.192 & $0.001[1.5 \mathrm{e}-$ & 0.225 \\
\hline & & {$[0.365 ; 0.867]$} & $4 ; 0.381]$ & $5 ; 0.769]$ & $5 ; 0.950]$ & {$[0.649 ; 0.956]$} & [0.054:0.378] & $4 ; 0.060]$ & [0.092:0.436] \\
\hline & PL & 0.207 & $8.7 \mathrm{e}-4[8.3 \mathrm{e}-$ & $0.001[7.5 \mathrm{e}-$ & $0.005[2.1 \mathrm{e}-$ & 0.208 & $0.002[2.5 \mathrm{e}-$ & $0.001[1.5 \mathrm{e}-$ & $0.005[7.8 \mathrm{e}-$ \\
\hline \multirow{6}{*}{$\begin{array}{l}\text { HEL } x \\
\text { BYN }\end{array}$} & SL & 12.724 & $0.008[5.9 \mathrm{e}-$ & $0.015[8.4 \mathrm{e}-$ & $0.001[4.4 \mathrm{e}-$ & 13.350 & $0.019[2.4 \mathrm{e}-$ & $0.003[1.5 \mathrm{e}-$ & $0.001[2.3 \mathrm{e}-$ \\
\hline & & {$[6.041 ; 14.941]$} & $5 ; 6.901]$ & $5 ; 13.919]$ & $6 ; 0.993]$ & {$[11.035 ; 15.521]$} & $4 ; 3.742]$ & $4 ; 2.425]$ & $5 ; 0.263]$ \\
\hline & $\mathrm{BD}$ & 0.798 & $0.002[5.4 \mathrm{e}-$ & $0.003[6.7 \mathrm{e}-$ & $0.003[5.8 \mathrm{e}-$ & 0.825 & 0.128 & $0.001[1.4 \mathrm{e}-$ & 0.148 \\
\hline & & {$[0.398 ; 0.945]$} & $5 ; 0.419]$ & $5 ; 0.846]$ & $5 ; 0.959]$ & {$[0.701 ; 0.989]$} & {$[0.028 ; 0.300]$} & $4 ; 0.112]$ & {$[0.041 ; 0.334]$} \\
\hline & PL & 0.725 & $0.002[5.3 \mathrm{e}-$ & $0.004[8.4 \mathrm{e}-$ & $0.003[6.4 \mathrm{e}-$ & 0.755 & 0.277 & $0.001[2.2 \mathrm{e}-$ & 0.382 \\
\hline & & {$[0.356 ; 0.848]$} & $5 ; 0.384]$ & $5 ; 0.762]$ & $5 ; 0.955]$ & {$[0.624 ; 0.918]$} & {$[0.149 ; 0.447]$} & $4 ; 0.060]$ & {$[0.236 ; 0.531]$} \\
\hline
\end{tabular}

Table 1. Summary statistics of the main quantitative genetics parameters in sticklebacks.

Estimates of the total phenotypic variance $\left(\mathrm{V}_{\mathrm{P}}\right)$, additive genetic variance $\left(\mathrm{V}_{\mathrm{A}}\right)$, dominance genetic variance $\left(\mathrm{V}_{\mathrm{D}}\right)$ and heritability $\left(h^{2}\right)$ from the animal model using the PRM or GRM. Posterior mode and 95\% highest posterior density (HPD; in brackets) are reported for standard length (SL), body depth (BD) and pelvic length (PL) for each dataset. All variance components were corrected following Legarra (2015; see Methods) to provide meaningful comparisons between models and datasets. 


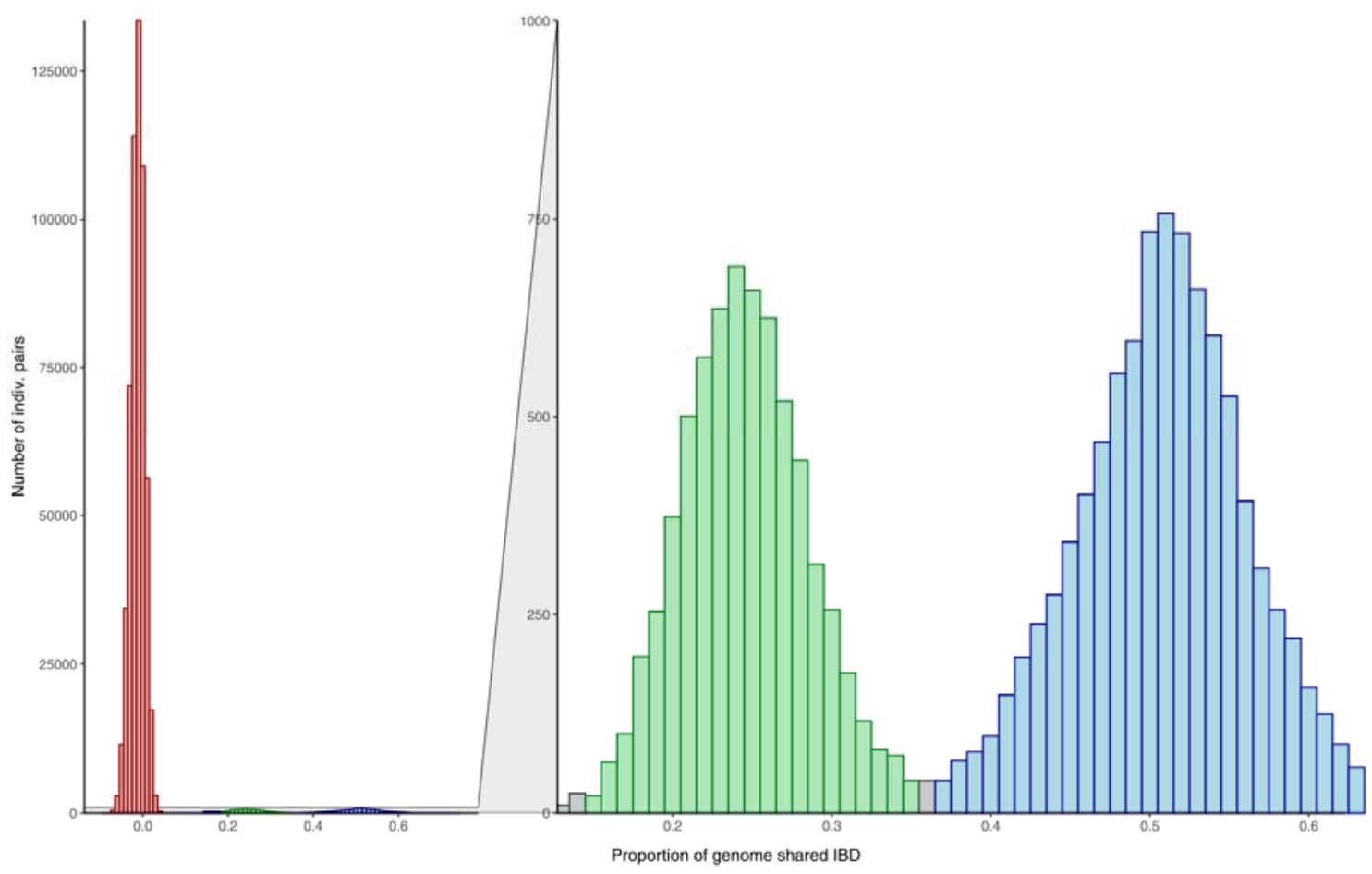

Figure 1. Distribution of IBD proportion in Helsinki crosses. The proportion of genome shared IBD among all pairs of individuals from the Helsinki crosses is shown among parents (red), half-sibs (green) and full-sibs (blue). Overlaps in IBD distributions are represented by grey shadings. 

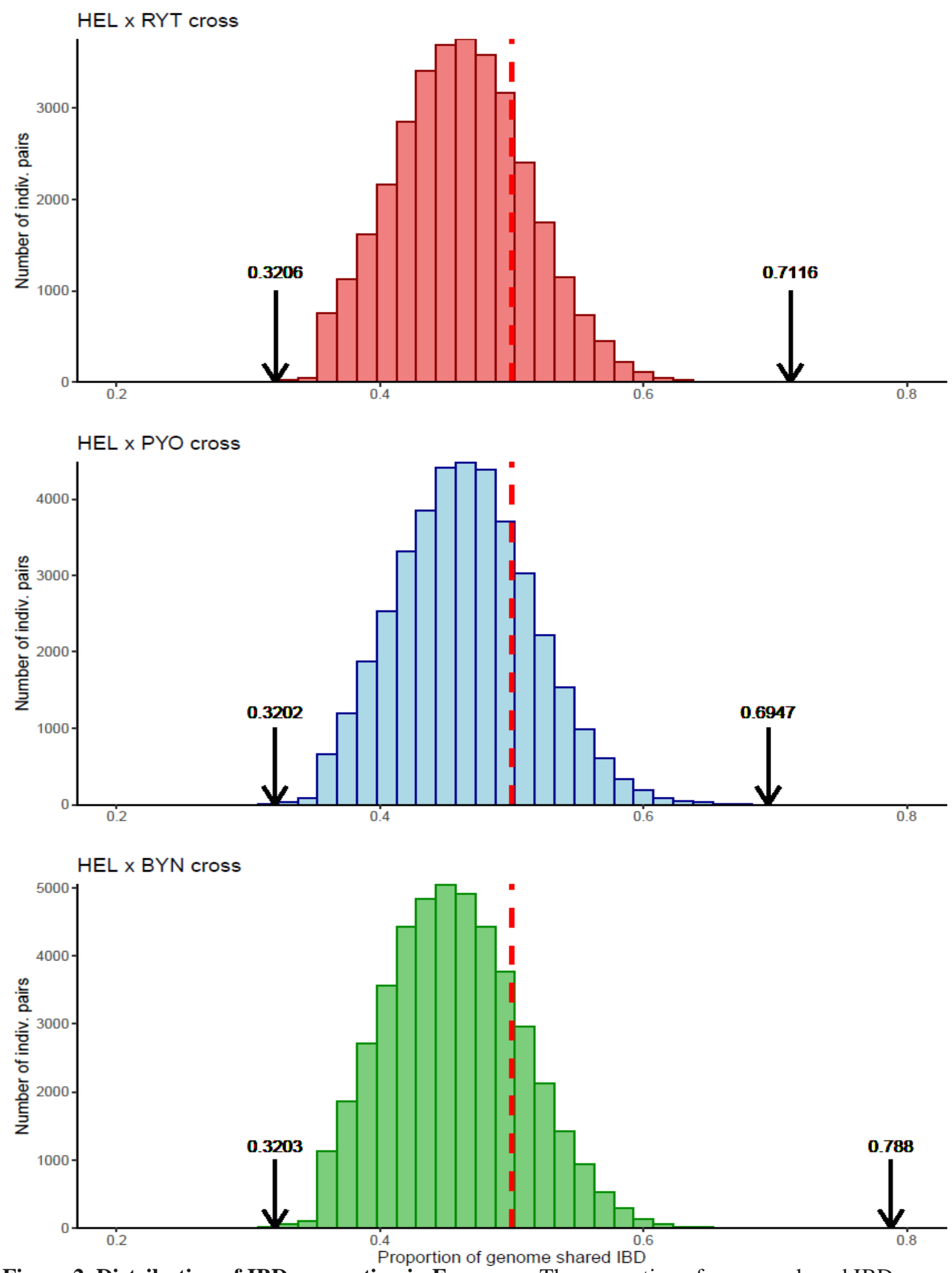

Figure 2. Distribution of IBD proportion in $\mathbf{F}_{2}$ crosses. The proportion of genome shared IBD among all pairs of individuals from the $\mathrm{F}_{2}$ crosses estimated with the KING MoM (Manichaikul et al. 2010). For each cross, the minimum and maximum values of relatedness coefficient are shown (black arrows). Red vertical dashed line depicts the theoretical 0.50 expectation of full-sibling relatedness. 

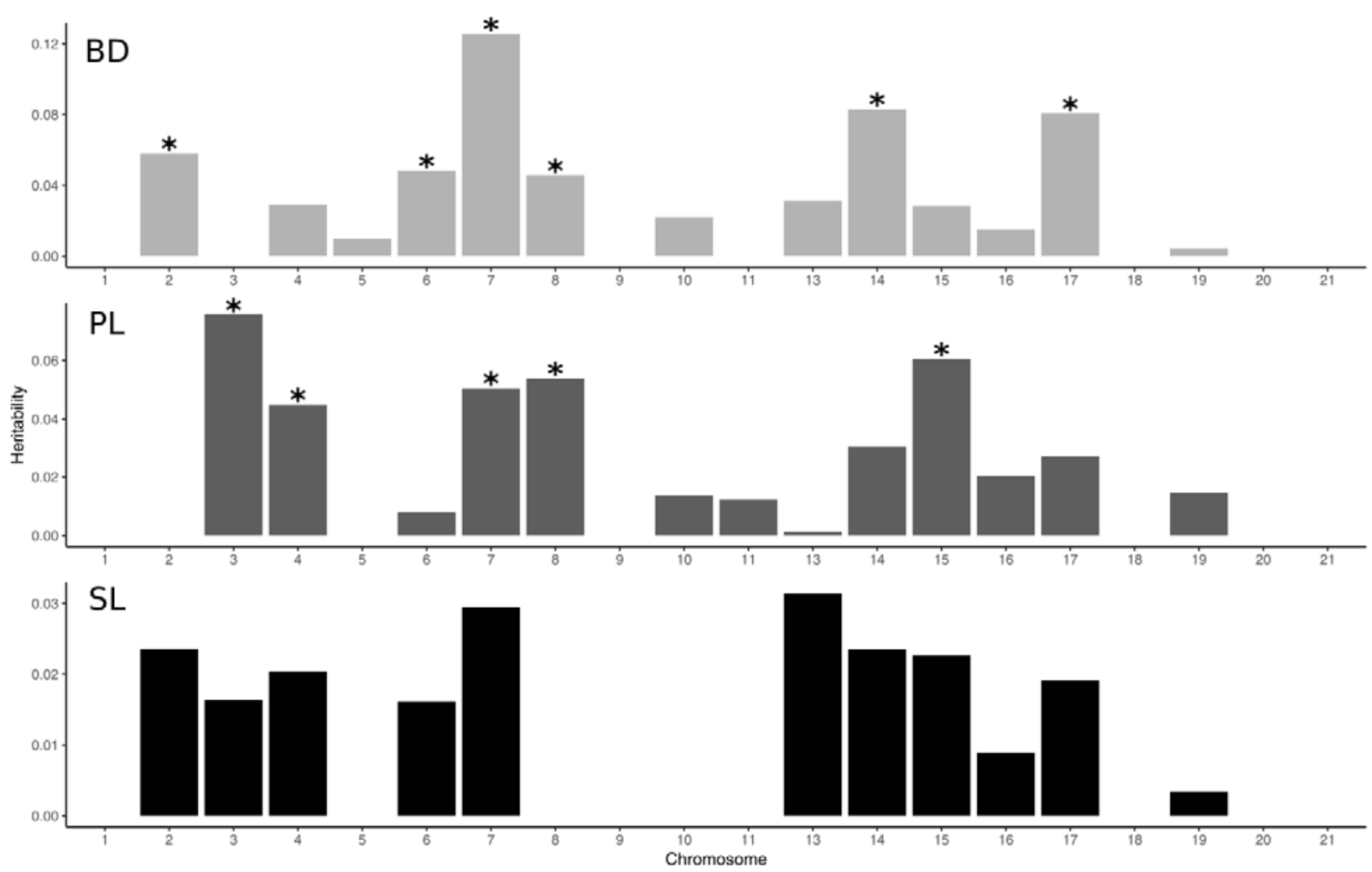

Figure 3. Chromosome partitioning of the genetic variance (Helsinki). The phenotypic variance in body depth (BD), pelvic spine length (PL) and standard length (SL) explained by the SNPs are shown of each chromosome for the Helsinki crosses. Black stars correspond to non-zero estimates (+/-SE) for each chromosome. 

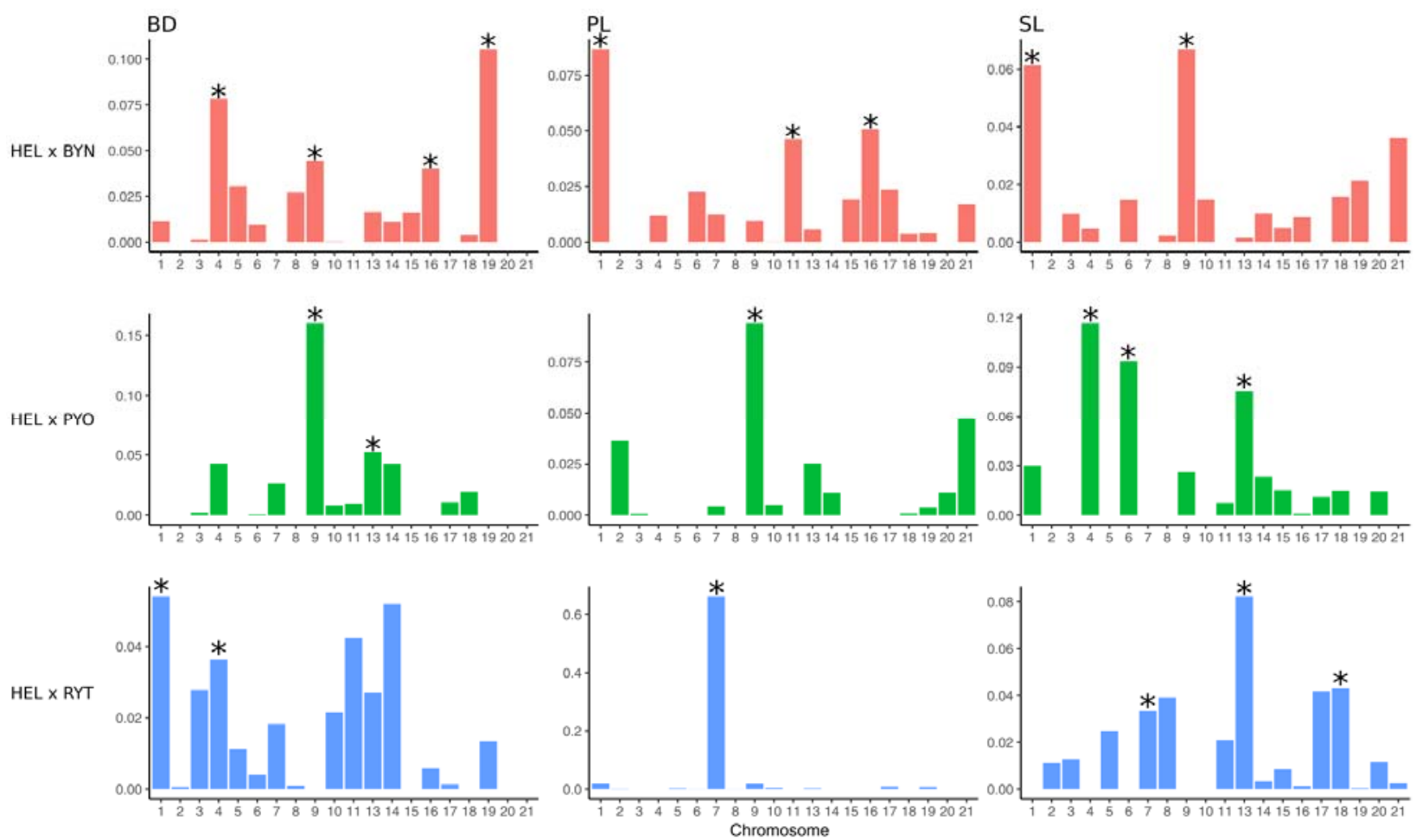

Figure 4. Chromosome partitioning of the genetic variance (QTL crosses). The phenotypic variance in body depth (BD; left column), pelvic spine length (PL; center column) and standard length (SL; right column) explained by the SNPs are shown of each chromosome for the HB cross (red; top row), HP cross (green; middle row) and HR (blue; bottom row). Black stars correspond to non-zero estimates (+/-SE) for each chromosome. 